\title{
The 9-Anthroate Chromophore as a Fluorescent Probe for Water
}

\author{
Antônio L. Maçanita, ${ }^{*}$ Fernando P. Costa, Sílvia M. B. Costa, Eurico C. Melo, and Helena Santos \\ Centro de Quimica Estrutural Complexo Interdisciplinar, Instituto Superior Técnico, Av. Rovisco Pais, \\ 1096 Lisboa Codex, Portugal (Received: February 1, 1988; In Final Form: May 6, 1988)
}

\begin{abstract}
Water quenches the fluorescence of methyl 9-anthroate with a rate constant showing little dependence on solvent viscosity or polarity. In dioxane, at $20^{\circ} \mathrm{C}$ the value of the rate constant is $9.6 \times 10^{6} \mathrm{M}^{-1} \mathrm{~s}^{-1}$, and the activation energy found for the process is $14.1 \mathrm{~kJ} \mathrm{~mol}^{-1}$. The quenching process is entropy-controlled and is likely to involve a hydrogen-bonded complex as an intermediate. Since the fluorescence lifetime of methyl 9-anthroate does not depend on the solvent properties other than its hydrogen-bonding ability, the concentration of nearby water can be estimated directly. Values of 3,54 , and $14 \mathrm{M}$ were obtained for the solubilization site of methyl 9-anthroate in micelles of Triton X-100, sodium dodecyl sulfate (SDS), and dodecyltrimethylammonium chloride (DTAC), respectively. From the ring current effect of the anthroate group on the ${ }^{1} \mathrm{H}$ NMR chemical shifts of the surfactant protons, it is concluded that the anthroate fluorescent probe is preferentially located in the surface region of the SDS and DTAC micelles; however, in Triton X-100, it resides in the micelle interior near the phenoxy groups of the surfactant molecule.
\end{abstract}

\section{Introduction}

During the past two decades considerable effort has been made on the study of micelles regarding their fluidity ${ }^{1-6}$ and polarity. ${ }^{7-13}$ Many of these studies were performed employing fluorescent probes, i.e., molecules whose fluorescence spectrum, quantum yield, or lifetime depends on one of the above properties in a convenient manner. ${ }^{14}$

Although polarity probes have been used to evaluate the water content in micelles, ${ }^{8-14}$ a probe that responds directly to the presence of water (i.e., not through polarity) has not yet been described as such. In those reports, the response to the polarity of the surrounding medium of either the energy of the fluorescence or the fluorescence lifetime gave some qualitative information on the amount of water in the probe vicinity (in spite of the wellknown difficulties involved in the building up of a calibration curve for polarity ${ }^{14}$ because of the choice of the polarity parameter and the set of solvents). Specifically, it is difficult to separate the polarizability effects from those of the dipole moment or hydrogen bonding on the spectroscopic property under study (spectrum or lifetime).

Several years ago, Werner et al. pointed out that the fluorescence of the 9-anthroate chromophore was strongly quenched in the presence of hydrogen-bond donors such as alcohols and water. ${ }^{15,16}$ This feature opens up the possibility of using this

(1) Blatt, E.; Ghiggino, K. P.; Sawyer, W. J. Chem. Soc., Faraday Trans. 1 1981, 77, 2551; J. Phys. Chem. 1982, 86, 4461. 771 .

(2) Emert, J.; Beherens, C.; Goldberg, M. J. Am. Chem. Soc. 1979, 101,

(3) Pownall, H. J.; Smith, J. C. J. Am. Chem. Soc. 1973, 95, 3136.

(4) Blatt, E.; Chatelier, R. C.; Sawyer, W. H. Photochem. Photobiol. 1984, $39,477$.

(5) Zachariasse, K. A. Chem. Phys. Lett. 1978, 57, 429.

(6) Costa, S. M. B.; Macanita, A. L. J. Phys. Chem. 1980, 84, 2402.

(7) Zachariasse, K. A.; Kozankiewicz, B.; Kuhnle, W. In Surfactants in Solution; Mittal, K. L., Lindman, B., Eds.; Plenum: New York, 1984; Vol. $1, \mathrm{p} 564$.

(8) Dill, K. A.; Koppel, D. E.; Cantor, R. S.; Dill, J. D.; Bendedouch, D.; Chen, S. H. Nature (London) 1984, 309, 42.

(9) Wennerstrom, H.; Lindman, B. J. Phys. Chem. 1979, 83, 2931.

(10) Menger, F. M. Acc. Chem. Res. 1979, 12, 111.

(11) Bendedouch, D.; Chen, S. H.; Koehler, W. C. J. Phys. Chem. 1983, 87,153 .

(12) Blatt, E.; Ghiggino, K. P.; Sawyer, W. H. Chem. Phys. Lett. 1985, 114,47 .

(13) Ulmius, J.; Lindman, B.; Lindblom, G.; Drakenberg, T. J. Colloid Interface Sci. 1978, 65, 88.

(14) Zachariasse, K. A.; Van Phuc, N.; Kozankiewicz, B. J. Phys. Chem. $1981,85,2676$.

(15) Werner, T. C.; Hercules, D. M. J. Phys. Chem. 1969, 73, 2005.

(16) Werner, T. C.; Hoffman, R. M. J. Phys. Chem. 1973, 77, 1611. See also: Werner, T. C. In Modern Fluorescence Spectroscopy; Wehry, E. L., Ed.; Plenum: New York, 1976; Vol. 2. chromophore as a specific probe of the water content in molecular organizates provided the following additional requirements are fulfilled: (a) the probe must solubilize exclusively in the organized phase, (b) the mean location of the probe in the organizate must either be known or be determinable by an independent technique, (c) the fluorescence quantum yield or lifetime of the probe must be independent of the environment properties (e.g., fluidity, polarity, or polarizability) other than the water content, (d) the quenching by water should also be independent of the solvent properties, and (e) the probe should not introduce a great perturbation in the organizate.

The work reported here deals with the evaluation of the fulfillment of these conditions by the 9-anthroate chromophore. Namely, (1) the mechanism and the kinetics of the fluorescence quenching of methyl 9-anthroate by water are investigated in some detail, and (2) the fluorescence properties and the mean location of methyl 9-anthroate when incorporated in SDS, DTAC, and Triton X-100 micelles are studied making use of fluorescence and ${ }^{1} \mathrm{H}$ NMR techniques.

\section{Experimental Section}

Reactants and Sample Preparation. The synthesis of methyl 9-anthroate has been previously described. ${ }^{17}$

Dioxane (Merck Uvasol) was either dried over sodium or molecular sieves $4 \mathrm{~A}$ or used as purchased. Drying did not change the fluorescence of the samples. Water was distilled twice, SDS (Merck, biochemical grade) was recrystallized from ethanol, and Triton X-100 (BDH scintillation grade) was used as purchased.

The concentration of methyl 9-anthroate was kept at $5 \times 10^{-5}$ $M$ except for the samples used in the flash photolysis experiments and in the NMR experiments (see below). The concentration of surfactant was $0.1 \mathrm{M}$ in all cases. Micellar solutions were allowed to stand for at least $24 \mathrm{~h}$ before measurement. ${ }^{18}$ All solutions were degassed by the freeze-pump-thaw technique (six cycles at $10^{-4}$ Torr).

Apparatus and Methods. Fluorescence spectra were measured with an Hitachi Perkin-Elmer MPF-3 spectrofluorometer. The analogic output of the MPF-3 was digitized with a Keithley 163 digital voltmeter and stored in a PDP $11 / 23$ computer. The instrumental response at each wavelength was corrected by using fluorescence standards. ${ }^{19}$ The method was found to give better accuracy than the correction unit which is part of the MPF-3.

The fluorescence quantum yields of methyl 9-anthroate in dioxane and dioxane-water were measured with anthracene and quinine sulfate as standards, where $\phi_{0}$ of anthracene in ethanol

(17) Moore, J. A.; Reed, D. E. Org. Synth. 1961, 41, 16.

(18) Melo, E. C. C.; Costa, S. M. B. J. Phys. Chem. 1987, 91, 5635.

(19) Melhuish, W. H. Appl. Opt. 1975, 14, 26. 


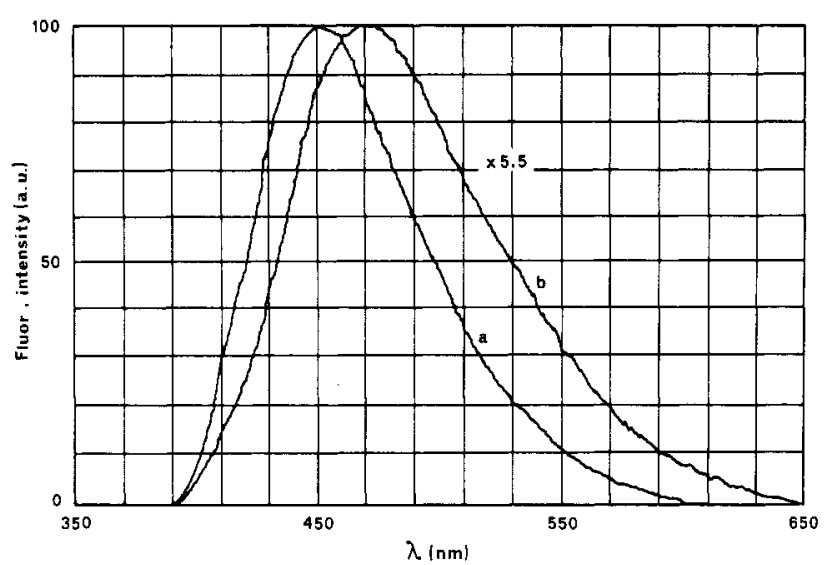

Figure 1. Emission spectra of methyl 9-anthroate in dioxane and in a dioxane-water $(26.4 \mathrm{M})$ mixture, at $20^{\circ} \mathrm{C}$. The last spectrum is multiplied by a factor of 5.5 .

equals $0.27^{20}$ and $\phi_{0}$ of quinine bisulfate in $1 \mathrm{~N} \mathrm{H}_{2} \mathrm{SO}_{4}$ equals $0.546{ }^{21}$ The integration of the spectra was made over the emission wavelength range, and corrections for changes in the refractive index were done according to Parker. ${ }^{22}$

Fluorescence decays were obtained by using the time-correlated single photon counting technique. The apparatus is identical with one previously described. ${ }^{23}$ The excitation was made with an Edinburgh Instruments lamp 199F filled with $\mathrm{N}_{2}\left(\lambda_{\text {exc }}=337 \mathrm{~nm}\right)$, and the emission of the sample was measured at $450 \mathrm{~nm}$ with a Philips XP2020Q photomultiplier. Alternate measurements $\left(10^{3}\right.$ counts at the maximum per cycle) of the pulse profile, at $337 \mathrm{~nm}$, and sample emission ${ }^{23}$ were performed until $2 \times 10^{4}$ to $3 \times 10^{4}$ counts at the maxima were reached. The decays in micellar solutions were measured with the emission polarizer set at the magic angle in order to depolarize the sample emission. The photomultiplier "wavelength shift" was evaluated from the best fits to a single exponential of the fluorescence decays of 9,10 diphenylanthracene in benzene, measured at emission wavelengths in the range between 420 and $500 \mathrm{~nm}$, with excitation at $337 \mathrm{~nm}$ $\left(1 \mathrm{ps} \mathrm{nm} \mathrm{nm}^{-1}\right.$ at $\left.2.35 \mathrm{kV}\right)$. The fluorescence decays were deconvoluted by using a program based on the modulation functions method as implemented by Striker, with shift correction ${ }^{24}$ (software package SANDBOX for Digital PDP 11/73 and VAX 780).

Triplet-triplet absorption spectra and decays were obtained with a conventional kinetic flash photolysis apparatus from Applied Photophysics. The flash energies were $200 \mathrm{~J}(\mathrm{fwhm}=4 \mu \mathrm{s})$. The concentration of methyl 9-anthroate was $1.5 \times 10^{-5} \mathrm{M}$ for all the samples. Under these conditions the initial triplet concentration is $10 \%$ of that of the ground state. The transients were stored in a Nicolet oscilloscope (Model 3091) transferred to the PDP $11 / 73$ and then fitted to a particular decay law, using standard nonlinear least-squares methods.

Preparative irradiations of degassed solutions of methyl 9anthroate were carried out with stirring using the $365-\mathrm{nm}$ line of a medium-pressure $\mathrm{Hg}$ arc lamp selected with appropriate chemical filters $\left(I(365) \approx 3 \times 10^{-7}\right.$ einstein $\left.\mathrm{s}^{-1}\right)$.

${ }^{1} \mathrm{H}$ NMR spectra were run in a Bruker CXP-300 spectrometer working at a frequency of $300.066 \mathrm{MHz}$ for protons. For each spectrum 32 scans were acquired and transformed with a digital resolution of $0.146 \mathrm{~Hz}$ point ${ }^{-1}$. A good digital resolution is essential because of the small magnitude of the expected ring current shifts. The sample was thermostated at $25 \pm 0.5^{\circ} \mathrm{C}$. The chemical shifts are referred to the HDO resonance at $4.76 \mathrm{ppm}$. Whenever

(20) Birks, J. B.; Dyson, D. J. Proc. R. Soc. London, A 1963, 275, 135. (21) Meech, S. R.; Phillips, D. J. Photochem. 1983, 23, 193.

(22) Parker, C. A. Photoluminescence of Solutions; Elsevier: Amsterdam, 1968.

(23) Zachariasse, K. A.; Duveneck, G.; Busse, R. J. Am. Chem. Soc. 1984, 106,1045 .

(24) Striker, G. In Deconvolution and Reconvolution of Analytical Signals; Bouchy, M., Ed.; DPIC: Nancy, France, 1982.
TABLE I: Refractive Indexes, $n^{20}$, of Dioxane-Water Mixtures, Fluorescence Quantum Yields, $\phi$, Lifetimes, $\tau$, and Radiative, $k_{f}$, and Nonradiative, $k_{\text {urr }}$, Rate Constants of Methyl 9-Anthroate in Dioxane and Dioxane-Water Mixtures at $20^{\circ} \mathrm{C}$

\begin{tabular}{cccccc}
\hline$\left[\mathrm{H}_{2} \mathrm{O}\right], \mathrm{M}$ & $n^{20}{ }_{\mathrm{D}}$ & $\phi$ & \multicolumn{1}{c}{$\mathrm{ns}$} & $k_{\mathrm{f}}{ }^{a} 10^{7} \mathrm{~s}^{-1}$ & $k_{\mathrm{nr}}{ }^{b} 10^{7} \mathrm{~s}^{-1}$ \\
\hline 0 & 1.4216 & 0.75 & 12.80 & 5.98 & 4.39 \\
4.4 & 1.4200 & 0.49 & 8.46 & 5.76 & 6.06 \\
8.8 & 1.4183 & 0.36 & 6.58 & 5.47 & 9.7 \\
13.2 & 1.4167 & 0.29 & 5.18 & 5.60 & 13.7 \\
17.6 & 1.4148 & 0.23 & 4.28 & 5.37 & 18.0 \\
22.0 & 1.4125 & 0.18 & 3.40 & 5.29 & 24.1 \\
26.4 & 1.4085 & 0.15 & 2.86 & 5.23 & 29.6 \\
${ }^{a} k_{\mathrm{f}}=\phi / \tau$. & ${ }^{b} k_{\mathrm{nr}}=(1-\phi) / \tau$. & & &
\end{tabular}

considered useful, the resolution of the spectra was enhanced by multiplication with a Gaussian function prior to Fourier transformation. In order to minimize the possible perturbation of the probe environment, the probe-to-surfactant ratio was $1 / 40$.

\section{Fluorescence Quenching of Methyl 9-Anthroate}

3.1. Results. Fluorescence in Dioxane-Water Mixtures. The fluorescence spectrum of methyl 9-anthroate in dioxane is structureless, with a maximum at $455 \mathrm{~nm}$. The lack of a mirror-image symmetry with the absorption spectrum (not shown) was attributed to the different configurations of the molecule in its ground (nonplanar) and first singlet excited (planar) states. ${ }^{15}$ Upon addition of water the fluorescence maximum shifts progressively toward longer wavelengths, reaching a value of 474.5 $\mathrm{nm}$ for $\left[\mathrm{H}_{2} \mathrm{O}\right]=26.4 \mathrm{M}$ (Figure 1). This large bathochromic shift is due to the large dipole moment of methyl 9-anthroate in the excited state $\left(\mu_{\mathrm{e}}-\mu_{\mathrm{g}}=5.2 \mathrm{D}\right)$, where the lowest $\pi \pi^{*}$ singlet state has a strong contribution from the anthracenecarboxylate charge-transfer configuration. ${ }^{25}$

A 5-fold decrease of the fluorescence quantum yield is observed between dioxane and the dioxane-water mixture with the largest water content (Table I). The fluorescence decays of methyl 9-anthroate in dioxane and in all dioxane-water mixtures are single exponentials. The decrease of the fluorescence lifetime with increase of water concentration is smaller than the one observed in the fluorescence quantum yield (Table I).

The radiative, $k_{\mathrm{f}}$, and the sum of the nonradiative rate constants $k_{\mathrm{nr}}=k_{\mathrm{ic}}+k_{\mathrm{isc}}$, where $k_{\mathrm{ic}}$ is the internal conversion rate constant and $k_{\text {isc }}$ is the intersystem crossing rate constant, were calculated from these data and are also given in Table $I$. The radiative rate constant, $k_{\mathrm{f}}$, is nearly unchanged, in agreement with earlier Werner's data. ${ }^{16}$ Nevertheless, a decreasing trend is observed upon addition of water. The refractive indexes of the mixtures decrease in the same manner.

It becomes apparent from the data given in Table I that the response of $\phi$ and $\tau$ to the presence of water is due to the variation of the sum of the nonradiative rate constants, $k_{\mathrm{nr}}$. A similar conclusion was previously reached by Werner et al. ${ }^{16}$

Triplet-Triplet Absorption. The T-T absorption spectrum of methyl 9-anthroate in dioxane, at $20^{\circ} \mathrm{C}$, is identical with respect to shape and wavelength maximum with the one observed in benzene $\left(\epsilon_{\mathrm{TT}}(432 \mathrm{~nm})=6.3 \times 10^{4} \mathrm{M}^{-1} \mathrm{~cm}^{-1}\right){ }^{26}$ The absorbance decays were monitored at $425 \mathrm{~nm}$ and fitted (Figure 2a) to decay kinetics including $\mathrm{T}-\mathrm{T}$ annihilation:

$$
\frac{1}{\left[{ }^{3} \mathrm{~A}^{*}\right](t)}=\frac{1}{\left[{ }^{3} \mathrm{~A}^{*}\right](0)} e^{t / \tau_{\mathrm{T}}}+k_{\mathrm{TT}} \mathrm{T}_{\mathrm{T}}\left(e^{t / \tau_{\mathrm{T}}}-1\right)
$$

In this equation, ${ }^{3} \mathrm{~A}^{*}$ represents the first triplet state of methyl 9 -anthroate, $\tau_{\mathrm{T}}$ is the triplet lifetime, and $k_{\mathrm{TT}}$ is the rate constant of triplet-triplet annihilation.

In neat dioxane, the best fit for eq 1 is obtained with $k_{\mathrm{TT}}=$ $1.2 \times 10^{8} \mathrm{M}^{-1} \mathrm{~s}^{-1}, \tau_{\mathrm{T}}=18 \mathrm{~ms}$, and $\left[{ }^{3} \mathrm{~A}^{*}\right](0)=1.7 \times 10^{-6} \mathrm{M}$

(25) Costa, S. M. B.; Macanita, A. L.; Prieto, M. J. J. Photochem. 1979 11,109 . The coefficient of the CT configuration in the $\mathrm{S}_{1}$ state is 0.37

(Maçanita, A. Ph.D. Thesis, Lisbon, 1980).
(26) Costa, S. M. B.; Melo, E. C. J. Chem. Soc., Faraday Trans. 2 1980,

76,1 

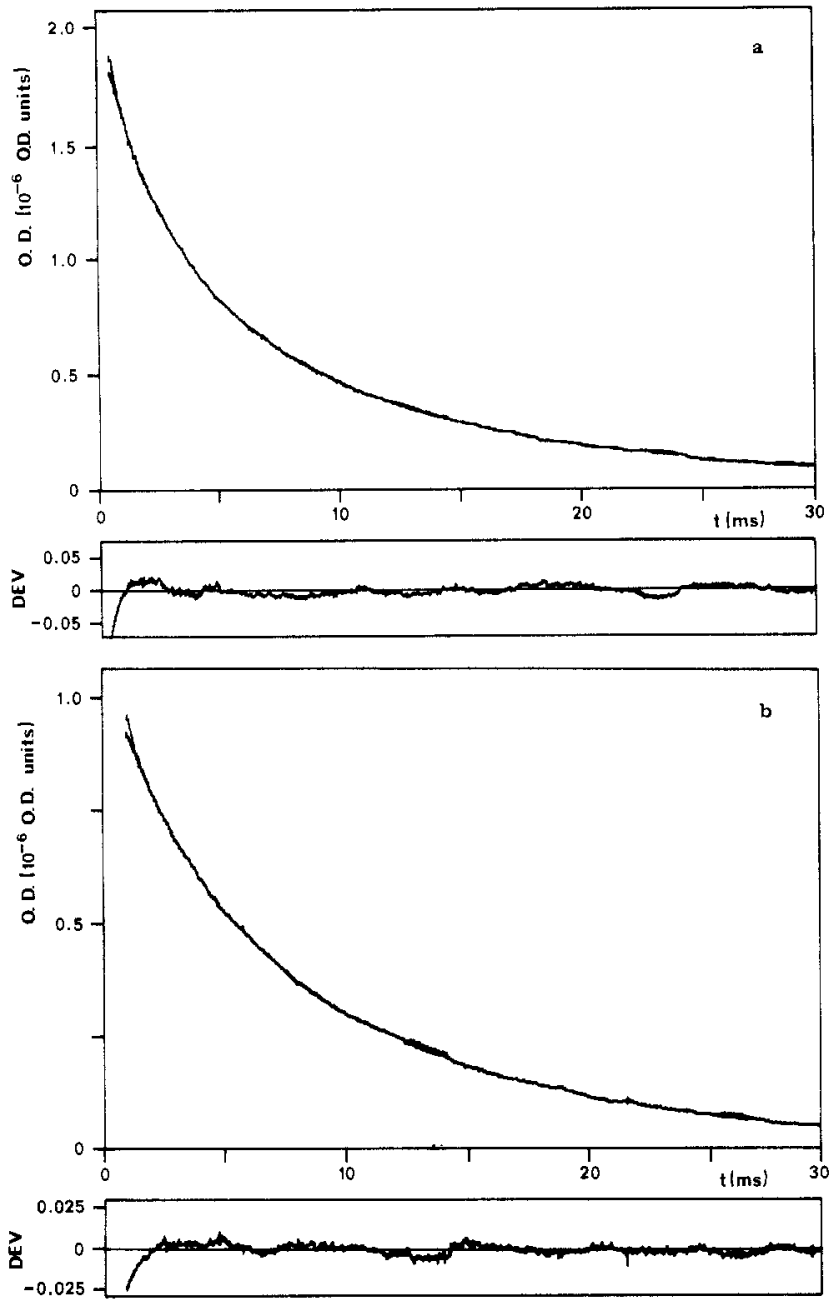

Figure 2. Triplet-triplet absorption decay of methyl 9-anthroate in (a) dioxane and (b) dioxane-water $(4.4 \mathrm{M})$.

(Figure $2 \mathrm{a}$ ). In a dioxane-water mixture, e.g., $4.4 \mathrm{M}$ in water (Figure $2 \mathrm{~b}$ ), $k_{\mathrm{TT}}$ has a similar value of $1.1 \times 10^{8} \mathrm{M}^{-1} \mathrm{~s}^{-1}, \tau_{\mathrm{T}}=$ $13 \mathrm{~ms}$, and $\left[{ }^{3} \mathrm{~A}^{*}\right]\left(\mathrm{H}_{2} \mathrm{O}\right)(0)=1.0 \times 10^{-6} \mathrm{M}$.

From these results two remarks should be stressed:

1. The small observed decrease in $\tau_{\mathrm{T}}$ shows that water does not efficiently quench the triplet state of methyl 9-anthroate $\left(k_{\mathrm{q}}\right.$ $\approx 10 \mathrm{M}^{-1} \mathrm{~s}^{-1}$ ).

2. The triplet concentration at time zero, $\left[{ }^{3} \mathrm{~A}^{*}\right](0)$, is 1.7 times smaller in the presence of water $(4.4 \mathrm{M})$ than the one obtained in neat dioxane. This factor is within the experimental error equal to the ratio of the fluorescence quantum yield in dioxane, $\phi$, and that observed with this mixture, $\phi_{0}\left(\phi_{0} / \phi=1.54\right)$. Thus, from the equation

$$
\frac{\left[{ }^{3} \mathrm{~A}^{*}\right](0)}{\left[{ }^{3} \mathrm{~A}^{*}\right]\left(\mathrm{H}_{2} \mathrm{O}\right)(0)}=\frac{k_{\text {isc }}}{k_{\text {isc }}\left(\mathrm{H}_{2} \mathrm{O}\right)} \frac{\phi_{0}}{\phi}
$$

one concludes that $k_{\text {isc }}$ does not depend on, or at most decreases with, the water content. This may be expected because the difference $E\left(\mathrm{~S}_{1}\right)-E\left(\mathrm{~T}_{1}\right)$ is rather large $(1.88 \mathrm{eV})$ and there is only one triplet state $\left(1.60 \mathrm{eV}\right.$, corresponding to the $T_{1}\left({ }^{3} \mathrm{~B}_{2 u}\right)$ state of anthracene) below $S_{1}(2.88 \mathrm{eV}){ }^{27}$

Therefore, the increase observed in the sum of the radiationless rate constants in the presence of water should be ascribed either to $k_{\text {ic }}$ or to some chemical or physical pathway with water being responsible for the quenching of the singlet state of methyl 9anthroate.

(27) Triplet-state energies were calculated by using the "advanced composite molecule" approach of the Parriser-Parr method with CI. The low energy of the $T_{1}\left(\pi, \pi^{*}\right)$ state explaines the difficulty in detecting the phosphorescence of methyl 9-anthroate (Macanita, A. L.; Melo, E. C.; Prieto, M. J., unpublished results).
TABLE II: Fluorescence Lifetimes, $\tau$, of Methyl 9-Anthroate in Solvents with Different Viscosities, $\eta$, and Dielectric Constants, $\epsilon$, at $20{ }^{\circ} \mathrm{C}$

\begin{tabular}{lccc}
\hline \multicolumn{1}{c}{ solvent } & $\eta^{20},{ }^{a} \mathrm{cP}$ & $\epsilon^{a}$ & $\tau, \mathrm{ns}$ \\
\hline$n$-pentane & 0.235 & 1.84 & 13.4 \\
$n$-hexane & 0.313 & 1.89 & 13.3 \\
$n$-heptane & 0.418 & 1.92 & 13.3 \\
methylcyclohexane & 0.734 & 2.02 & 12.9 \\
cyclohexane & 0.980 & 2.02 & 13.0 \\
$n$-hexadecane & 3.484 & & 13.3 \\
liq paraffin & 126 & & 13.0 \\
acetone & 0.324 & 20.7 & 12.5 \\
benzonitrile & 1.33 & 25.2 & $12.7^{b}$ \\
$N, N$-dimethylformamide & 0.924 & 36.7 & 12.8 \\
1,3-propanediol & 56.0 & 32.0 & 5.3 \\
ethanol & 1.20 & 24.3 & $4.1^{b}$ \\
methanol & 0.551 & 32.5 & $1.7^{b}$ \\
dioxane- $\mathrm{D}_{2} \mathrm{O}(11 \mathrm{M})$ & & & 6.2 \\
acetone- $\mathrm{H}_{2} \mathrm{O}(11 \mathrm{M})$ & & & 5.5 \\
1,3-propanediol- $\mathrm{H}_{2} \mathrm{O}(11 \mathrm{M})$ & & & 3.2
\end{tabular}

${ }^{a}$ From ref $34 .{ }^{b}$ At $22^{\circ} \mathrm{C}$, from ref 16 .

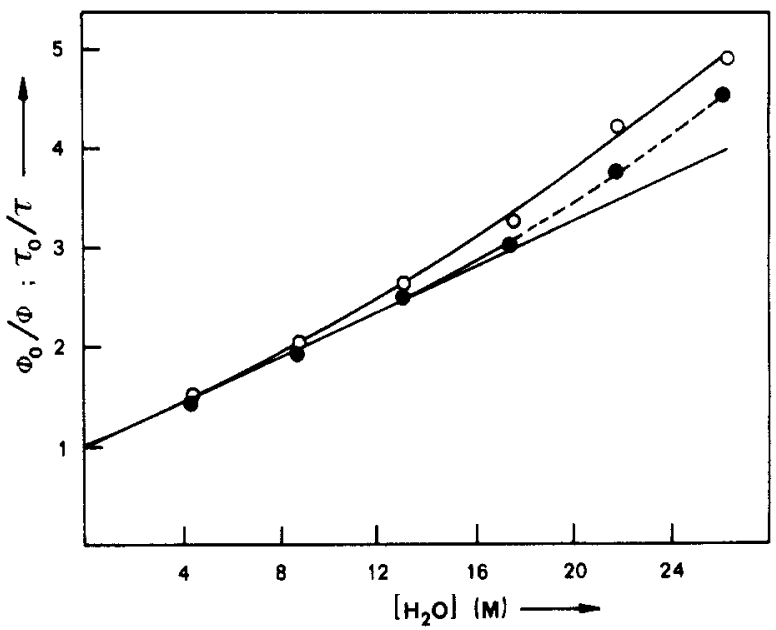

Figure 3. Stern-Volmer plots: $(O) \phi_{0} / \phi$ and $(\bullet) \tau_{0} / \tau$ in dioxane at 20 ${ }^{\circ} \mathrm{C}$.

Photochemical Reactivity. The photochemical reactivity of methyl 9-anthroate was investigated following its absorption spectra, fluorescence spectra, and fluorescence decays before and after irradiation (see Experimental Section).

Irradiation of a methyl 9-anthroate solution in a dioxane-water $(4.4 \mathrm{M})$ mixture, up to $3 \mathrm{~h}$, produced no changes in the spectra or the fluorescence decay, although during this time each molecule is excited an average of 20 times. The possible formation of the 9-anthroic acid was investigated because its spectrum and decay are similar to those of methyl 9-anthroate. Addition of $\mathrm{NaOH}$ to the irradiated samples produced no signs of the fluorescence spectrum of the 9-anthracenecarboxylate anion which is structured and blue-shifted with respect to its protonated form. ${ }^{15}$ Moreover, the fluorescence decays remained single exponential. These results exclude a chemical reaction with water as responsible for the observed quenching. Therefore, it is concluded that water quenches the methyl 9-anthroate singlet state through a physical process, namely, the internal conversion channel.

Fluorescence Lifetime as a Function of the Solvent Viscosity and Polarity. The fluorescence lifetimes of methyl 9-anthroate in several solvents at $20^{\circ} \mathrm{C}$ obtained from single-exponential decays are presented in Table II. The data of Werner for the lifetimes of methyl 9-anthroate in alcohols ${ }^{16}$ are also given in the same table. It is seen that the lifetimes are independent of the solvent viscosity and polarizability (solvents $1-7$ in Table II). In nonprotic solvents, also the solvent polarity does not induce any appreciable change on those decay times (solvents 8-10 in Table II). The fluorescence lifetime of methyl 9-anthroate in acetonitrile is decreased down to ca. $8 \mathrm{~ns}$, but this is due to a photochemical reaction of the ester with this solvent. ${ }^{28}$ Acetonitrile also reacts 


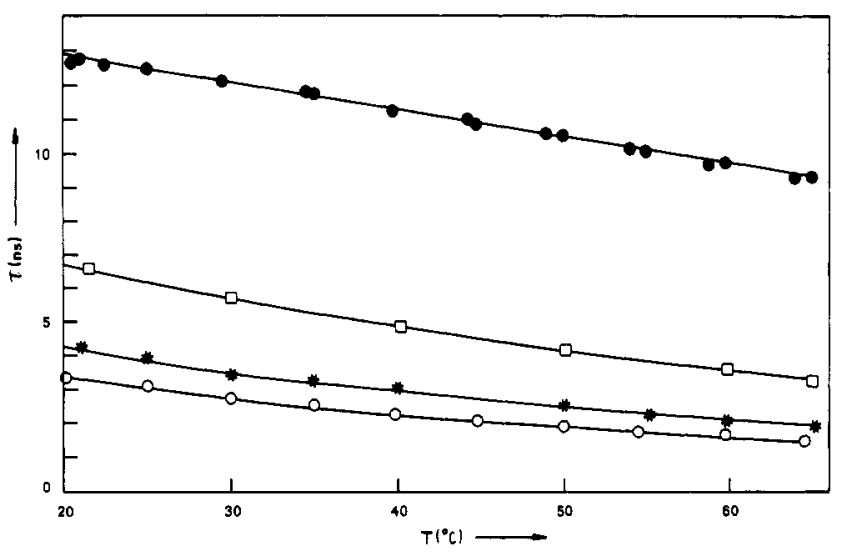

Figure 4. Fluorescence lifetimes of methyl 9 -anthroate in dioxane and dioxane-water mixtures, (ㅁ) $8.8 \mathrm{M},\left(^{*}\right) 17.6 \mathrm{M}$, and (O) $22.0 \mathrm{M}$, as a function of temperature.

with other aromatic esters. ${ }^{25}$ Therefore, the quenching process is exclusively due to the presence of $\mathrm{OH}$ groups (see bottom of Table II).

Quenching Rate Constant. The ratios of the fluorescence quantum yields of methyl 9-anthroate, in the absence and in the presence of water, $\phi_{0}$ and $\phi$, respectively, and also the corresponding fluorescence lifetime ratios, $\tau_{0} / \tau$, are plotted against water concentration in Figure 3. It is seen that the Stern-Volmer plots $^{29}$ are nonlinear. This observation will be commented upon in the Discussion section.

For concentrations of water lower than ca. $20 \mathrm{M}$, the representation of $\tau_{0} / \tau$ vs $\left[\mathrm{H}_{2} \mathrm{O}\right]$ is linear, and the value of the quenching rate constant, $k_{\mathrm{a}}=9.6 \times 10^{6} \mathrm{M}^{-1} \mathrm{~s}^{-1}$, is obtained from the expression

$$
\tau_{0} / \tau=1+k_{\mathrm{a}} \tau_{0}\left[\mathrm{H}_{2} \mathrm{O}\right]
$$

This value is 3 orders of magnitude lower than the calculated diffusion-controlled rate constant in the dioxane-water mixtures which range from $7 \times 10^{9}$ to $1 \times 10^{10} \mathrm{M}^{-1} \mathrm{~s}^{-1}$.

The value of $k_{\mathrm{a}}$ (of water) in other solvents displaying viscosities and polarities different from those of dioxane can be evaluated with eq 3 (from the data in Table II). In acetone, at $20^{\circ} \mathrm{C}$ ( $\eta$ $=0.324 \mathrm{cP}, \epsilon=20.7$ ), the quenching rate constant has a value of $k_{\mathrm{a}}=9.0 \times 10^{6} \mathrm{M}^{-1} \mathrm{~s}^{-1}$, and in 1,3-propanediol $(\eta=46.6 \mathrm{cP}$, $\epsilon=35.0)$ the value is only slightly larger $\left(k_{\mathrm{a}}=1.1 \times 10^{7} \mathrm{M}^{-1}\right.$ $\left.\mathbf{s}^{-1}\right)$. Both values are similar to the one obtained in dioxane $(\eta$ $=1.30 \mathrm{cP}, \epsilon=2.2$ ), which shows that the 9-anthroate-water interaction depends neither on the solvent viscosity nor on the polarity. Furthermore, the fluorescence quenching by deuteriated water in dioxane $\left(k_{\mathrm{a}}=7.6 \times 10^{6} \mathrm{M}^{-1} \mathrm{~s}^{-1}\right)$ is less efficient than the one induced by $\mathrm{H}_{2} \mathrm{O}$.

This ensemble of results shows that the quenching of methyl 9-anthroate by water is reaction-controlled and it is strictly related to the formation of a hydrogen bond.

The ratios of the quantum yields are significantly greater than $\tau_{0} / \tau$. Larger values of $\phi_{0} / \phi$ than those of $\tau_{0} / \tau$ have been found either when transient effects on diffusion-controlled reactions are present $^{30,31}$ or when ground-state association leading to static quenching occurs. ${ }^{32,33}$ Since $k_{\mathrm{a}}$ is 3 orders of magnitude lower than the diffusion-controlled limit, transient effects can be ruled out. Ground-state association will next be investigated as a possible cause for the differences between $\phi_{0} / \phi$ and $\tau_{0} / \tau$.

(28) Chandross, E. A.; Thomas, H. T. Chem. Phys. Lett. 1971, 9, 393. Beddard, G. S.; Carlin, S. E.; Lewis, C. J. Chem. Soc., Faraday Trans 2 1975, 71,1894 .

(29) Stern, O.; Volmer, M. Phys. Z. 1919, 20, 183

(30) Nemzek, T. L.; Ware, W. R. J. Chem. Phys. 1975, 62, 477.

(31) Costa, S. M. B.; Macanita, A. L.; Formosinho, S. J. J. Phys. Chem. $1984,88,4089$.

(32) Ware, W. R. Pure Appl. Chem. 1975, 41, 635

(33) Schroeder, J.; Troe, J. Chem. Phys. Lett. 1985, 116, 453.

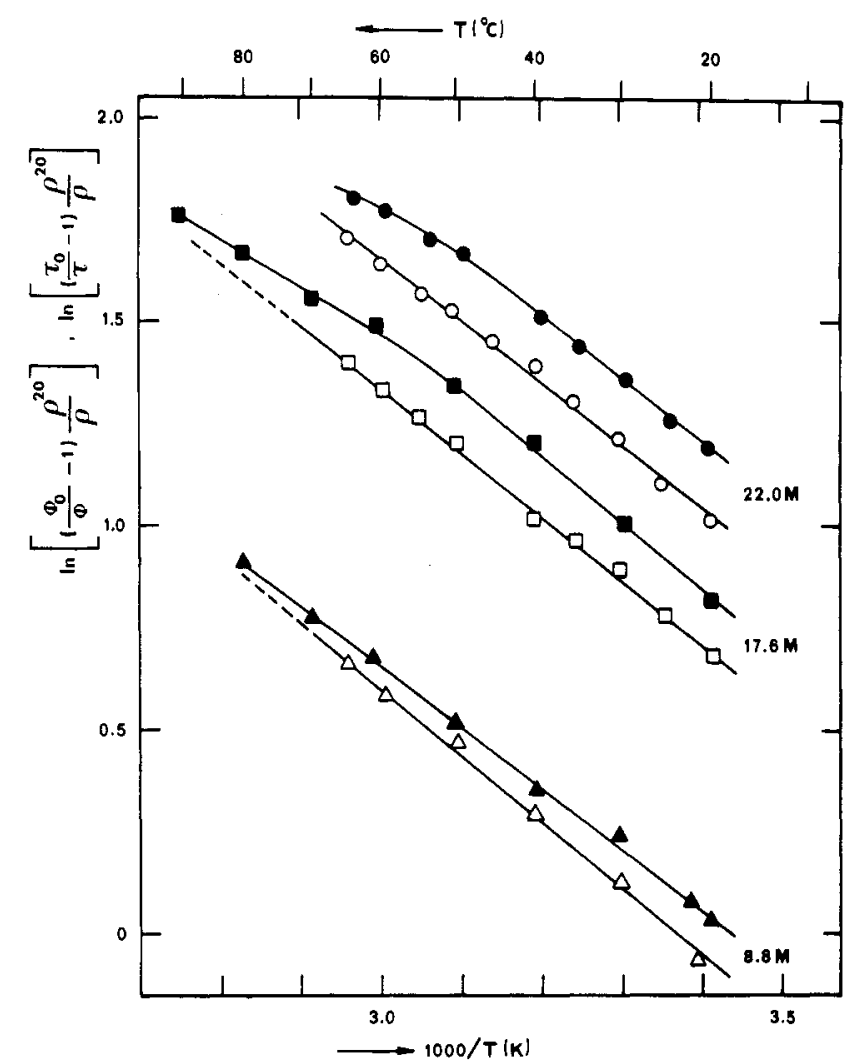

Figure 5. Arrhenius plot from steady-state $\left(\left(\phi_{0} / \phi-1\right) \rho^{20} / \rho^{T}\right)$ (filled symbols) and time-resolved $\left(\left(\tau_{0} / \tau-1\right) \rho^{20} / \rho^{T}\right)$ (empty symbols) data for three concentrations of water: $8.8,17.6$, and $22.0 \mathrm{M}$.

Fluorescence Decays and Quantum Yields as a Function of Temperature. The fluorescence quantum yield and the lifetime of methyl 9-anthroate in dioxane and in dioxane-water mixtures $\left(\left[\mathrm{H}_{2} \mathrm{O}\right]=8.8,17.6\right.$, and $\left.22.0 \mathrm{M}\right)$ were measured as a function of the temperature $\left(20-80^{\circ} \mathrm{C}\right.$ ) (Figure 4).

The Arrhenius plot of the quenching rate constant, $k_{\mathrm{a}}$, which is assumed to be proportional to $\left(\tau_{0} / \tau-1\right) \rho^{20} / \rho^{T}$ (see eq 3 ), is shown in Figure 5. $\left(\rho^{20} / \rho^{T}\right.$ corrects for the change in water concentration due to the variation of the density with temperature.) Values of the activation energy $E_{\mathrm{a}}=-R \mathrm{~d}\left(\ln k_{\mathrm{a}}\right) / \mathrm{d}(1 / T)=14.1$ $\mathrm{kJ} \mathrm{mol}{ }^{-1}$ and the preexponential factor $k_{\mathrm{a}}{ }^{0}=3 \times 10^{9} \mathrm{M}^{-1} \mathrm{~s}^{-1}$ are obtained from this plot. These values are identical within the experimental accuracy for the three concentrations of water (Figure 5). Since, as shown before, the quenching process is reaction-controlled ( $k_{\mathrm{a}}$ is independent of solvent viscosity and is much smaller than the diffusion-controlled rate constants in those solvents), $E_{\mathrm{a}}$ and $k_{\mathrm{a}}{ }^{0}$ refer to the quenching step itself free from diffusional contributions. Within this context, it should be noted that $k_{\mathrm{a}}$ is low due to a small preexponential factor and not to a large activation energy.

In Figure 5 , plots of $\ln \left[\left(\phi_{0} / \phi-1\right) \rho^{20} / \rho^{T}\right]$ as a function of the reciprocal temperature are also shown. These plots present a downward curvature due to a gradual decrease of the differences between $\phi_{0} / \phi$ and $\tau_{0} / \tau$. At about $90^{\circ} \mathrm{C}$ the difference becomes negligible for $\left[\mathrm{H}_{2} \mathrm{O}\right]$ equal to 8.8 and $17.6 \mathrm{M}$. These observations are compatible with an exothermic ground-state association between methyl 9-anthroate and water. With the increase of temperature, the equilibrium is shifted toward the free species and, therefore, the difference between $\phi_{0} / \phi$ and $\tau_{0} / \tau$ decreases. At the high-temperature limit, where the concentration of the associated ester is negligible, $\phi_{0} / \phi$ equals $\tau_{0} / \tau$.

3.2. Discussion. Ground-State Association. The ground-state association of methyl 9-anthroate with water, due to hydrogen

(34) Rossini, F.; Pitzer, K.; Arnett, R.; Braun, R.; Pimentel, G. Selected Values of Physical and Thermodynamic Properties of Hydrocarbons and Related Compounds; Carnegie: Pittsburg, 1953; pp 226-235. Maryott, A. A.; Smith, E. R. "Table of Dielectric Constants of Pure Liquids"; Natl. Bur. Stand. (U.S.) Circ. 1951, No. 514. 


\section{SCHEME I}

$$
\begin{aligned}
& \mathrm{A}+\mathrm{H}_{2} \mathrm{O} \rightleftharpoons \mathrm{KB}=\mathrm{H}-\mathrm{O}-\mathrm{H} \\
& \text { a. } I_{0}|\quad|(1-a) I_{0} \\
& { }^{1} A^{*}+\mathrm{H}_{2} \mathrm{O} \stackrel{k_{2}}{\longrightarrow}{ }^{1} A^{*} \ldots H-\mathrm{O}-\mathrm{H} \\
& 1 / \tau_{0} \downarrow
\end{aligned}
$$

bonding between the two species, is anticipated. Evidence for this association was previously obtained by using the FT-IR technique (Fourier transform infrared spectroscopy) to monitor the stretching vibration of the anthroate carbonyl group $\left(1726 \mathrm{~cm}^{-1}\right)$. Upon addition of water, a new absorption band at lower energies (1710 $\mathrm{cm}^{-1}$ ), which is attributable to the hydrogen-bond complex, is observed. ${ }^{35}$ From a plot of the integrated intensity of these two bands as a function of the water concentration, the value of the equilibrium constant of this complex at $20^{\circ} \mathrm{C}$ was obtained to be $K_{\mathrm{b}}=1 \times 10^{-2} \mathrm{M}^{-1}$. 35 This means that, e.g., for the mixture $17.6 \mathrm{M}$ in $\mathrm{H}_{2} \mathrm{O}, 15 \%$ of methyl 9-anthroate is hydrogen-bonded to water. (The absence of evidence for hydrogen bonding in the electronic absorption spectrum is due to the noncoplanarity of the carboxylate and the anthracene ring in the ground state.)

The difference between $\phi_{0} / \phi$ and $\tau_{0} / \tau$ can be rationalized if the hydrogen-bonded fraction of the excited molecules is quenched immediately after the excitation time. The simplest kinetic scheme compatible with the above considerations and the spectroscopic data involves four species: the free anthroate in the ground and in the excited states and the hydrogen-bonded complex also in the ground and in the excited states (Scheme I).

The dissociation of the excited hydrogen-bonded complex back to the excited methyl 9-anthroate is neglected on the basis of the observed single exponentiality of the decays of methyl 9-anthroate at any emission wavelength and temperature; i.e., $1 / \tau_{0}{ }^{\prime}$ is much larger than the dissociation rate constant.

In Scheme I, A represents the anthroate chromophore, $K_{\mathrm{b}}$ is the equilibrium constant of association in the ground state, $\alpha$ is the fraction of the absorbed light, $I_{0}$, due to $\mathrm{A}$, which is related to $K_{\mathrm{b}}$ through eq $4,1 / \tau_{0}$ is the reciprocal lifetime of ${ }^{1} \mathrm{~A}^{*}$ in the absence of quenching, and $k_{\mathrm{a}}$ is the rate constant of association in the excited state.

$$
\alpha=\frac{1}{1+K_{\mathrm{b}}\left[\mathrm{H}_{2} \mathrm{O}\right]}
$$

According to this scheme, the concentration of ${ }^{1} \mathrm{~A}^{*}$ as a function of time follows an exponential law:

$$
\left[{ }^{1} \mathrm{~A}^{*}\right]=\left[{ }^{1} \mathrm{~A}^{*}\right]_{0} e^{-t / \tau}
$$

The reciprocal decay parameter, $1 / \tau$, is given by eq 6 , which can be rearranged to yield the modified Stern-Volmer equation (eq 3).

$$
\frac{1}{\tau}=\frac{1}{\tau_{0}}+k_{\mathrm{a}}\left[\mathrm{H}_{2} \mathrm{O}\right]
$$

Besides, under photostationary state conditions, the following equation is obtained.

$$
\begin{aligned}
\phi_{0} / \phi= & \left(1+k_{\mathrm{a}} \tau_{0}\left[\mathrm{H}_{2} \mathrm{O}\right]\right)\left(1+K_{\mathrm{b}}\left[\mathrm{H}_{2} \mathrm{O}\right]\right) \\
& =\left(\tau_{0} / \tau\right)\left(1+K_{\mathrm{b}}\left[\mathrm{H}_{2} \mathrm{O}\right]\right)
\end{aligned}
$$

From a plot of $\left(\phi_{0} / \phi\right) /\left(\tau_{0} / \tau\right)$ vs $\left[\mathrm{H}_{2} \mathrm{O}\right]$ (Figure 6), a value of $K_{\mathrm{b}}$ $=0.7 \times 10^{-2} \mathrm{M}^{-1}$, at $20^{\circ} \mathrm{C}$, is calculated in reasonable agreement with the one obtained from FT-IR experiments $\left(1 \times 10^{-2} \mathrm{M}^{-1}\right)$.

The temperature dependence of $\left(\phi_{0} / \phi\right) /\left(\tau_{0} / \tau\right)$ for three dioxane-water mixtures is shown in Figure 7 . From the three van't Hoff plots shown, the same value of the association enthalpy, $\Delta H_{\mathrm{b}}{ }^{\circ}$ $=-4.2 \mathrm{~kJ} \mathrm{~mol}^{-1}$, is obtained

(35) Villalain, J. Gomez-Fernandez, J, C.; Prieto, M. J. J. Colloid Interface Sci. 1988, 124, 233. Abstracts of Papers, 3rd European Conference on the Spectroscopy of Biological Molecules, Reims, France, 1985; Manfait, M., Alix, A. J. P., Bernard, L., Eds.; Wiley: New York, 1985; pp 296-298.

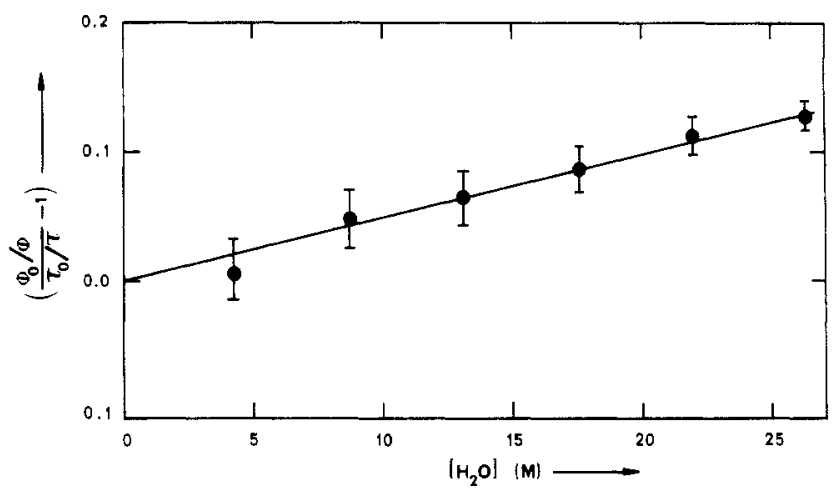

Figure 6. Plot of $\left\{\left[\left(\phi_{0} / \phi\right) /\left(\tau_{0} / \tau\right)\right]-1\right\}$, at $20^{\circ} \mathrm{C}$, as a function of water concentration.

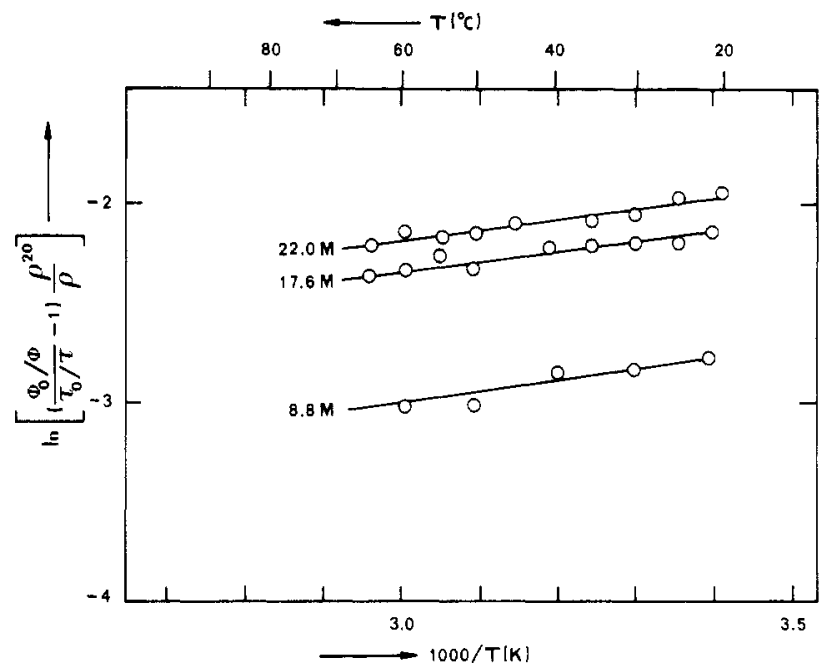

Figure 7. Plot of $\ln \left\{\left[\left(\phi_{0} / \phi\right) /\left(\tau_{0} / \tau\right)\right]-1\right\} \rho^{20} / \rho^{T}$ vs the reciprocal temperature for three concentrations of water: $8.8,17.6$, and $22.0 \mathrm{M}$

Mechanism. The activation enthalpy, $\Delta H^{\ddagger}$, and the activation entropy, $\Delta S^{*}$, at $20^{\circ} \mathrm{C}$, for the anthroate-water interaction in dioxane can be calculated with eq 8 and 9 , where $h, k$, and $R$ are the Planck, Boltzmann, and gas constants, respectively, and $e$ is the basis of natural logarithms.

$$
\begin{gathered}
\Delta H^{*}=E_{\mathrm{a}}-R T \\
\Delta S^{*}=R\left(\ln k_{\mathrm{a}}^{0}-\ln \frac{k T}{h} e\right)
\end{gathered}
$$

Their values are $\Delta H^{*}=11.7 \mathrm{~kJ} \mathrm{~mol}^{-1}$ and $\Delta S^{*}=-72 \mathrm{~J} \mathrm{~mol}^{-1}$ $\mathrm{K}^{-1}$ (standard state $c^{\circ}=1 \mathrm{M}$ ), showing that $k_{\mathrm{a}}$ is small due to a large entropy change involved in the quenching process. This entropy control indicates a large stereospecificity of the quenching step which, in turn, is consistent with the hydrogen-bond formation. Consequently, since the radiative and the intersystem crossing rate constants do not depend on the presence of water, it is concluded that the fluorescence quenching of methyl 9 -anthroate by water is due to an efficient radiationless decay of the hydrogen-bond complex. This conclusion suggests that it is specifically the hydrogen bond of water to the carboxylate group of the anthroate molecule that provides the coupling mode to the solvent states which provides the efficient radiationless process of methyl 9anthroate. The assumption also explains the absence of significant quenching of the $\pi, \pi^{*}$ triplet state, where the carboxylate group is twisted with respect to the anthracene ring. ${ }^{36}$ In this configuration the carboxylate and therefore the hydrogen bond are not coupled to the anthracene $\pi$ system. This has two consequences: (1) the deactivation process will become inefficient, and (2) the charge density on the oxygen atoms becomes small (no chargetransfer contribution), making the hydrogen bond weak (smaller $K_{\text {b). }}$.

(36) Melo, E. C. Ph.D. Thesis, Lisbon, 1987. 
Kinetics. The upward curvature of the Stern-Volmer plot obtained from photostationary data $\left(\phi_{0} / \phi-1\right)$ is due to the ground-state anthroate-water association (see eq 7). However, the nonlinearity of the $\left(\tau_{0} / \tau-1\right)$ plot is not compatible with eq 3 and deserves further discussion. This curvature leads to a small variation of $k_{\mathrm{a}}$ from $9.6 \times 10^{6} \mathrm{M}^{-1} \mathrm{~s}^{-1}$, at low $\left[\mathrm{H}_{2} \mathrm{O}\right]$, to $1.13 \times$ $10^{7} \mathrm{M}^{-1} \mathrm{~s}^{-1}$ at the highest concentration, which can in principle be due to three causes: (a) more complex kinetics than the one of Scheme I, (b) increase of $k_{\mathrm{a}}$ with the polarity of the medium, and (c) decrease of $\tau_{0}$ with the polarity of the medium besides the one derived from the hydrogen-donating ability of the solvent.

(a) Complex Kinetics. Scheme I does not explicitly contain the equilibria of water self-association or dioxane-water association. Consequently, the effective concentration of water, $\left[\mathrm{H}_{2} \mathrm{O}\right]$, is in fact different from the analytical concentration, $\left[\mathrm{H}_{2} \mathrm{O}\right]_{0}$. The inclusion, in Scheme I, of one single equilibrium (eq 10) induces

$$
\begin{gathered}
\mathrm{H}_{2} \mathrm{O}+\mathrm{H}_{2} \mathrm{O} \stackrel{K_{\text {dim }}}{\rightleftarrows} \mathrm{H}_{2} \mathrm{O} \cdots \mathrm{HOH} \\
{ }^{1} \mathrm{~A}^{*}+\mathrm{H}_{2} \mathrm{O} \cdots \mathrm{HOH} \stackrel{k_{\mathrm{a}}^{\text {dim }}}{\longrightarrow} \text { quenching }
\end{gathered}
$$

a significant increase on the complexity of the kinetics; namely, two extra unknowns, $K_{\text {dim }}$ and $k_{\mathrm{a}}^{\text {dim }}$, are introduced. However, introduction of steps 10 and 11 would lead to a downward curvature of the Stern-Volmer plot (see eq 12; Appendix), in contrast with the experimental observation.

$$
\left[\mathrm{H}_{2} \mathrm{O}\right]=\frac{\left(1+8 K_{\mathrm{dim}}\left[\mathrm{H}_{2} \mathrm{O}\right]_{0}\right)^{1 / 2}-1}{4 K_{\mathrm{dim}}}
$$

(b) Increase of $k_{\mathrm{a}}$ with Polarity. The activation energies, $E_{\mathrm{a}}$, and the preexponential factors determined with three dioxanewater mixtures with different dielectric constants [8.8 $\mathrm{M}(\epsilon=8)$, $17.5 \mathrm{M}(\epsilon=19)$, and $22 \mathrm{M}(\epsilon=27)]^{37}$ have similar values within experimental accuracy. This fact indicates that the polarity does not induce major changes on $k_{\mathrm{a}}$. On the other hand, the slight increase of $k_{\mathrm{a}}$ from dioxane $\left(k_{\mathrm{a}}=9.6 \times 10^{6} \mathrm{M}^{-1} \mathrm{~s}^{-1}\right)$ to $1,3-$ propanediol $\left(k_{\mathrm{a}}=1.13 \times 10^{7} \mathrm{M}^{-1} \mathrm{~s}^{-1}\right)$ may be due to the increase of the solvent polarity.

(c) Decrease of $\tau_{0}$. The fluorescence lifetime of methyl 9anthroate in nonprotic solvents, $\tau_{0}$, is not sensitive to the polarity or to the viscosity of the solvent (see Results). Nevertheless, it cannot be excluded that, in addition to the quenching mechanism via hydrogen bonding, large concentrations of water may further reduce the fluorescence lifetime of methyl 9-anthroate.

In conclusion, factors $\mathrm{b}$ and $\mathrm{c}$ may affect the value of $k_{\mathrm{a}}$, although to a small extent; the observed variation of $k_{\mathrm{a}}(9.6 \times$ $10^{6}-1.13 \times 10^{7} \mathrm{M}^{-1} \mathrm{~s}^{-1}$ ) is lower than $10-20 \%$.

Thermodynamics. From the preceding discussion (factor a) it is clear that $K_{\mathrm{b}}$ in Scheme I is a pseudoequilibrium constant which also contains the equilibrium constants of the water-water and water-dioxane association processes. Therefore, the value of $\Delta H_{\mathrm{b}}{ }^{\circ}$, obtained from the variation of $\left[\left(\phi_{0} / \phi\right) /\left(\tau_{0} / \tau\right)\right]-1$ with the temperature, not only is the enthalpy change involved in the water-anthroate hydrogen-bonding process but also contains contributions from the enthalpy change associated with the water-water and water-dioxane hydrogen-bond breaking $\left(\Delta H_{\mathrm{b}}{ }^{0}\right.$ $=-13-15 \mathrm{~kJ} \mathrm{~mol}^{-1}$ for the water-dioxane hydrogen bond in $\mathrm{CCl}_{4}{ }^{38}$ ). These contributions decrease the absolute value of $\Delta H_{\mathrm{b}}{ }^{\circ}$, making it less negative than the formation enthalpy of the water-anthroate hydrogen-bond complex formed with the free species. Consequently, the observed value $\Delta H_{\mathrm{b}}{ }^{\circ}=-4.2 \mathrm{~kJ} \mathrm{~mol}^{-1}$ does not have a simple direct relationship with the strength of the anthroate-water hydrogen bond in the ground state.

From the preceding discussion the following conclusions can be derived: (1) The fluorescence lifetime of methyl 9-anthroate does not depend on solvent properties other than its hydrogenbonding ability. (2) The rate constant of fluorescence quenching by water is also independent of solvent polarity or viscosity.

(37) King, E. J. Acid-Base Equilibria; Pergamon: Oxford, 1965; p 252. (38) Vinogradov, S.; Linneli, R. Hydrogen Bonding, Van Nostrad Reinhold: New York, 1971; p 121

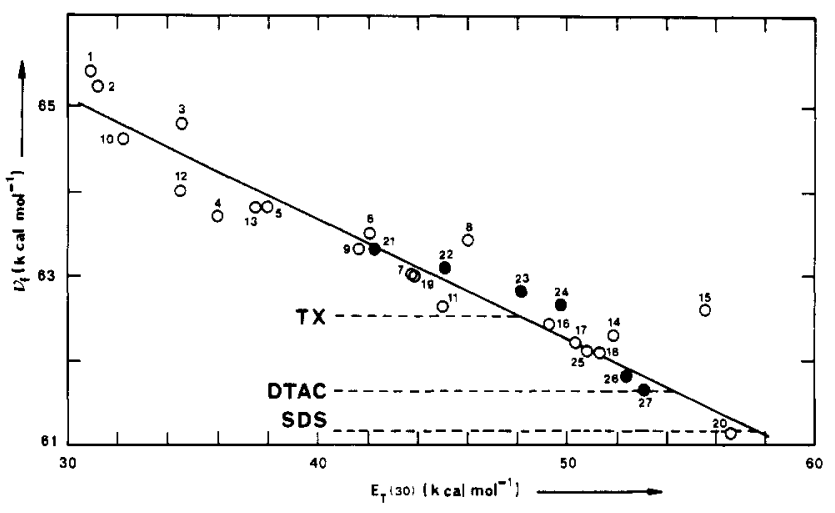

Figure 8. Energy of the intensity maximum in the uncorrected fluorescence spectra of methyl 9 -anthroate, at $20^{\circ} \mathrm{C}$, as a function of $E_{\mathrm{T}}(30)$ in (1) $n$-hexane, (2) cyclohexane, (3) diethyl ether, (4) dioxane, (5) ethyl acetate, (6) acetone, (7) $N, N$-dimethylformamide, (8) acetonitrile, (9) dichloromethane, (10) carbon tetrachloride, (11) dimethyl sulfoxide, (12) benzene, (13) tetrahydrofuran, (14) ethanol, (15) methanol, (16) 2propanol, (17) 1-butanol, (18) 1-propanol, (19) 2-methyl-2-propanol, (20) ethylene glycol, and (21-27) dioxane-water mixtures $\left(\left[\mathrm{H}_{2} \mathrm{O}\right]=2.2\right.$, $4.4,8.8,13.3,17.8,22.2,26.4 \mathrm{M}) . E_{\mathrm{T}}(30)$ values for the dioxane-water mixtures were calculated with the Langhals equation. ${ }^{39}$

TABLE III: Fluorescence Quantum Yields, $\phi$, Lifetimes, $\tau$, of Methyl 9-Anthroate in Dioxane, Triton X-100, SDS, and DTAC Micellar Solutions, at $25^{\circ} \mathrm{C}$, and the Concentration of Water and the Ground-State Equilibrium Constant, $K_{\text {by }}$ in the Solubilization Site in These Micelles

\begin{tabular}{lcccl}
\hline \multicolumn{1}{c}{ solvent } & $\phi$ & $\tau, \mathrm{ns}$ & {$\left[\mathrm{H}_{2} \mathrm{O}\right], \mathrm{M}$} & $K_{\mathrm{b}}, \mathrm{M}^{-1}$ \\
\hline dioxane & 0.72 & 12.4 & & 0.007 \\
Triton X-100 & 0.25 & 9.2 & 2.8 & 0.40 \\
DTAC & 0.14 & 4.6 & 14 & 0.06 \\
SDS & 0.051 & 1.6 & 54 & 0.015
\end{tabular}

Therefore, a direct determination of the concentration of water in the vicinity of the 9-anthroate chromophore should be possible, and consequently, this molecule can be considered as a suitable fluorescent probe for water. This assumption was submitted to experimental verification and is described in the next section.

\section{Determination of Water Concentration at the Solubilization Site of Methyl 9-Anthroate in SDS, DTAC, and Triton X-100 Micelles}

4.1. Results. Fluorescence Spectra. Methyl 9-anthroate is sparingly soluble in water, ${ }^{6}$ but it can be easily incorporated in SDS, DTAC, and Triton X-100 micelles. The absorption and the emission spectra in these micelles are red-shifted with respect to those in nonpolar solvents. The shift in the emission spectrum is particularly large in the case of SDS, as is seen in Figure 8, where the energies of the peak are compared with those found in neat solvents and dioxane-water mixtures covering a range of $E_{\mathrm{T}}(30)$ values. The values of $E_{\mathrm{T}}(30)$, corresponding to the solubilization site of methyl 9-anthroate in Triton X-100, DTAC, and SDS, taken from this calibration curve, are 48.0, 54.1, and $57.6 \mathrm{kcal} \mathrm{M}^{-1}$, respectively. In dioxane-water mixtures these values correspond to water concentrations of $8.4,27$, and $40 \mathrm{M}$, according to Langhals' data. ${ }^{39}$

Fluorescence Quantum Yields and Lifetimes. The fluorescence decays of methyl 9-anthroate in all three micelles are single exponential, provided they are measured with the emission polarizer set at the magic angle. The fluorescence lifetimes and quantum yields, at $25^{\circ} \mathrm{C}$, are presented in Table III. Using the value of $k_{\mathrm{a}}$ at $25^{\circ} \mathrm{C}\left(1.0 \times 10^{7} \mathrm{M}^{-1} \mathrm{~s}^{-1}\right)$ and that of the unquenched lifetime of methyl 9-anthroate in dioxane, at $25^{\circ} \mathrm{C}(12.3 \mathrm{~ns})$, in eq 3 , we calculated the concentrations of water in the vicinity of the probe in each micelle, and their values are also given in Table III $(2.8,15$, and $54 \mathrm{M}$ in Triton X-100, DTAC, and SDS). In SDS, the resulting value corresponds to the concentration of bulk water while, in Triton X-100, it is very small, indicating a rather

(39) Langhals, H. Angew. Chem. 1982, 94, 739. 


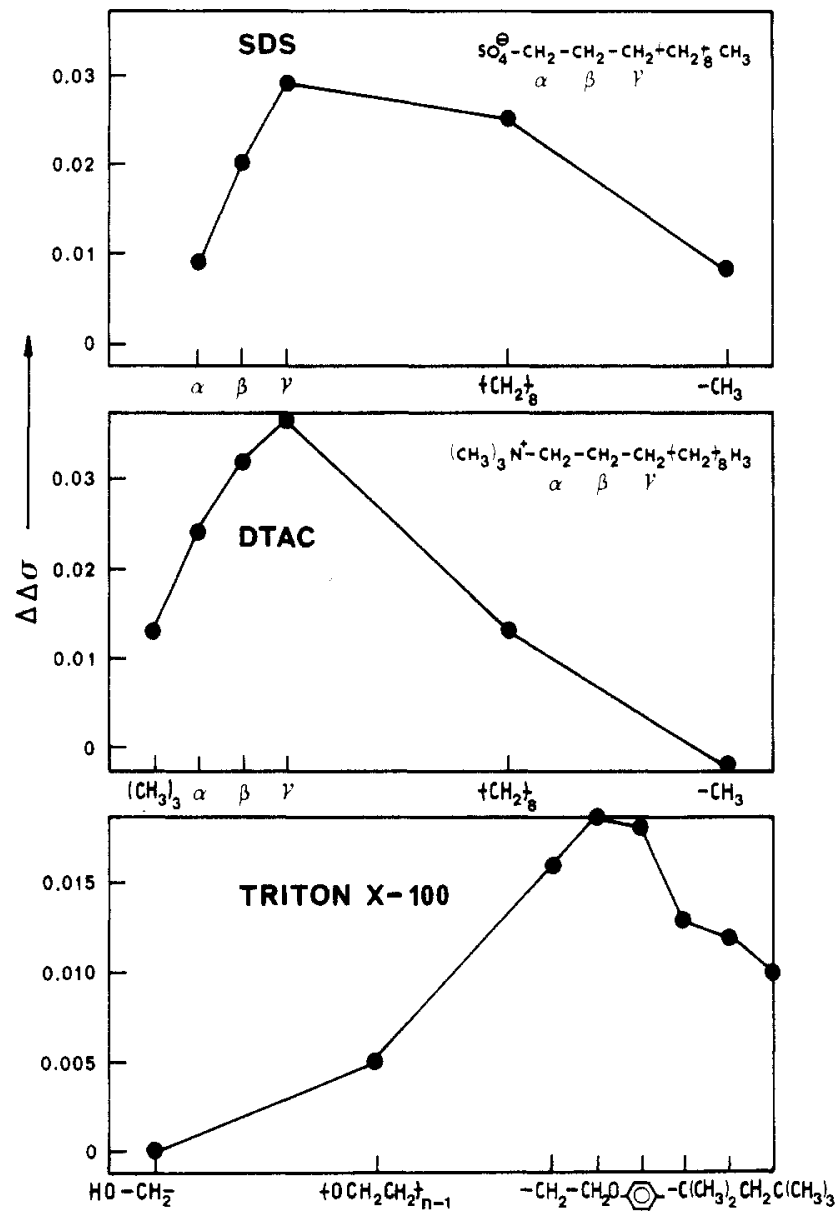

Figure 9. Difference, $\Delta \Delta \sigma$, on the NMR chemical shift of the SDS DTAC, and Triton X-100 protons in micelles, in the absence and in the presence of methyl 9-anthroate, at $25^{\circ} \mathrm{C}$. The assignments for the Triton $\mathrm{X}-100$ resonances are taken from ref 45

hydrophobic solubilization site of methyl 9-anthroate in this micelle.

The data in Table III (quantum yields and water concentrations) can be further used to evaluate the equilibrium constant $K_{\mathrm{b}}$ in the solubilization site of methyl 9-anthroate in these micelles, using eq 7 (see Table III). It is interesting to note that, while in SDS $K_{\mathrm{b}}$ is similar to the equilibrium constant in dioxane $\left(K_{\mathrm{b}}\right.$ $\left.=0.7 \times 10^{-2} \mathrm{M}^{-1}\right)$, this is not the case in Triton X-100 where a much larger value is found $\left(K_{\mathrm{b}}=0.40 \mathrm{M}^{-1}\right)$. The observation is compatible with the larger hydrophobicity of the location site in Triton X-100, where the lack of the water-water hydrogen bonds will lead to a larger equilibrium constant (see section 3.2).

Probe Location Using ${ }^{1} H N M R$. The localization of an aromatic hydrocarbon in a micelle can be evaluated from the ring current effects on the chemical shift of the resonances arising from the different protons of the surfactant molecule. This useful technique was introduced by Eriksson and Gillberg ${ }^{40}$ and extensively applied by Zachariasse et al. ${ }^{7,14,41}$

Micelles of Triton X-100 $\left[\left(\mathrm{CH}_{3}\right)_{3} \mathrm{CCH}_{2}\left(\mathrm{CH}_{3}\right)_{2}-\mathrm{Ph}-\right.$ $\left.\mathrm{OCH}_{2} \mathrm{CH}_{2}-\left(\mathrm{OCH}_{2} \mathrm{CH}_{2}\right)_{7,5}-\mathrm{OCH}_{2} \mathrm{CH}_{2} \mathrm{OH}\right]$ produce a well-resolved ${ }^{1} \mathrm{H}$ NMR spectrum enabling the specific identification of the chain segments near which the probe is located. However, both in DTAC and in SDS micelles, the protons of eight out of the eleven methylene groups have very similar resonance frequencies, giving a broad structureless resonance, which prevents a detailed characterization of the probe perturbation in a long region of the chain.

The differences in chemical shift $(\Delta \Delta \sigma)$ between the NMR spectra of SDS, DTAC, and Triton X-100 micelles, with and

(40) Eriksson, J. C.; Gillberg, G. Acta Chem. Scand. 1966, 20, 2019.

(41) Zachariasse, K. A.; Kozankiewicz, B.; Kuhnle, W. In Photochemistry and Photobiology; Zewail, A. H., Ed.; Harwood: London, 1983; Vol II. without methyl 9-anthroate, are plotted for the various proton resonances in Figure 9. The presence of the probe induces upfield shifts in all the protons except in the terminal methyl group of DTAC, suggesting that this group is not located over the aromatic ring. ${ }^{42}$ A similar downfield shift of the methyl group resonance has been previously reported for pyrene derivatives solubilized in CTAB and TTAB micelles. ${ }^{41}$

From the plots in Figure 9, it is apparent that the ring current effect is maximal on the external protons $(\alpha, \beta$, and the $\gamma$ methylene groups) of the SDS and DTAC micelles, suggesting that methyl 9-anthroate is predominantly located at the surface region (terminal polar groups plus a few carbons) of the SDS or DTAC micelles. In contrast with this, in Triton X-100 micelles, the most shifted resonances are those due to the aromatic protons and to the adjacent ethoxy group, indicating a probe location near the surface of the inner hydrophobic core and away from the aqueous surface.

In spite of the general conclusion that the probe is located at the surface of both SDS and DTAC micelles, the plots in Figure 9 for these two surfactants show quite different features. In DTAC micelles a sharp decrease of the probe ring current effect is observed for the chain segments next to the methylene group, while the plot obtained with SDS micelles suggests a broader distribution of effects.

4.2. Discussion. Water Concentrations from Fluorescence Quenching. From the fluorescence quenching data, water concentrations of $2.8,15$, and $54 \mathrm{M}$ were found in the vicinity of the solubilization site of methyl 9-anthroate in Triton X-100, DTAC, and SDS micelles, respectively. The last value $(54 \mathrm{M})$ deserves a lower confidence level than those obtained in Triton X-100 or DTAC, because the quenching kinetic study in dioxane was not extended to the concentration range near that of neat water due to the low solubility of methyl 9 -anthroate. In fact, water concentrations were calculated with the quenching rate constant value of $1.0 \times 10^{7} \mathrm{M}^{-1} \mathrm{~s}^{-1}$, which is valid up to $30 \mathrm{M}$ water concentration but is probably too low at $54 \mathrm{M}$. (See in section 3.1 the upward curvature of the $\tau^{0} / \tau$ Stern-Volmer plot.)

The ${ }^{1} \mathrm{H}$ NMR data showed that methyl 9-anthroate is located in the surface region of both SDS and DTAC micelles, but it solubilizes in the hydrophobic core of the Triton X-100 micelle, near the phenoxy groups. In this latter micelle, the value of water concentration at the solubilization site of the probe $(2.8 \mathrm{M})$ is much smaller than those reported in the literature $(29 \mathrm{M}$ for Triton $\mathrm{X}-100$ from X-ray scattering ${ }^{43}$ or $30 \mathrm{M}$ for pentaethylene glycol dodecyl ether from $\mathrm{NMR}^{44}$ ), which is understandable since these values refer to the mean water concentration in the whole micelle, while $2.8 \mathrm{M}$ refers specifically to the surface region of the inner core. The result shows that there is very little water in this region, which is in agreement with the qualitative conclusion of Podo et al. ${ }^{45}$ In addition, it also shows that methyl 9-anthroate does not draw an appreciable amount of water into the micelle core which is different from other probes containing polar groups. ${ }^{9}$

In both SDS and DTAC micelles, large concentrations of water (54 and $14 \mathrm{M}$, respectively) are obtained in the vicinity of the probe. Although the concentration in SDS is much larger than the one obtained in DTAC, which is compatible with the larger bathochromic shift of the emission spectrum in the SDS micelles, the NMR data indicate a probe location at the surface of both micelles. Particularly in the case of DTAC, only the resonances arising from the surface protons are affected (see Figure 9), which implies that the detection of a smaller concentration is not due to a deeper mean location of the probe in this micelle.

As pointed out before, the NMR data in SDS indicate a broad distribution of effects along the surfactant chain, all proton resonances being considerably perturbed. Considering that the probe "sees" bulk water in this micelle, this result must mean that, rather than a deeper location of the probe, part of the terminal methyl

(42) Podo, F.; Blaisie, J. K. Proc. Natl. Acad. Sci. U.S.A. 1977, 74, 1032

(43) Paradies, H. J. Phys. Chem. 1980, 84, 899.

(44) Nilsson, P. G.; Lindman, B. J. Phys. Chem. 1983, 87, 4756.

(45) Podo, F.; Ray, A.; Nemethy, G. J. Am. Chem. Soc. 1973, 95, 6164. 
and the middle methylene groups of the surfactant are located close to the surface. In fact, a linear arrangement of all the SDS monomers in the micelle is stereochemically impossible. ${ }^{46}$ Consequently, in order to rationalize the differences in the water content in the surface region of the SDS and DTAC micelles, more information is necessary, namely, data obtained with functional probes (e.g., the $n$-(9-anthroyloxy) stearic acids) with which different radial positions may be attained.

Water Concentrations from Fluorescence Spectra. Water concentrations can also be evaluated from the probe fluorescence spectra, if it is assumed that dioxane-water mixtures can be used to translate the $E_{\mathrm{T}}(30)$ values of the solubilization site of methyl 9-anthroate in micelles (Figure 8 ) into concentrations of water. When the values obtained in this way $(8.4,27$, and $40 \mathrm{M}$ in Triton X-100, DTAC, and SDS, respectively) are compared with those obtained from fluorescence quenching, qualitative agreement between the two methods is observed. Nevertheless, the "polarity method" gives water concentrations in Triton X-100 and DTAC which are respectively 3 - and 2-fold larger than those obtained from the "quenching method". This is not surprising because the shift in the emission spectrum of methyl 9-anthroate depends on all the dielectric properties of the medium, including polarizability, while the fluorescence lifetime is primarily dependent on the concentration of water.

\section{Conclusions}

From the previous results it is concluded that methyl 9-anthroate is a good fluorescent probe for water. This is due to the following reasons:

1. The fluorescence lifetime of methyl 9-anthroate is strongly quenched in the presence of water, and it does not depend on the solvent properties other than the hydrogen-bonding ability.

2. The quenching rate constant is also independent of the solvent which allows the extrapolation of its value, obtained in homogeneous media, to the micellar medium, i.e., a quantitative analysis of the results.

3. The mean location site of methyl 9-anthroate in micelles can be ascertained with the ${ }^{1} \mathrm{H}$ NMR technique.

4. The experiments can be performed with a small probe-tosurfactant molar ratio, i.e., a small perturbation (if any) of the micelle.

5. The necessary experimental data (fluorescence lifetimes) can be easily obtained with high accuracy.

The importance of this conclusion is reinforced by the fact that the 9-anthroate chromophore can be linked to different positions

(46) Chachaty, C. Prog. Nucl. Magn. Reson. Spectrosc. 1987, 19, 183. in fatty acid chains. Many of these compounds (e.g., the ( $n$ anthroyloxy) stearic acids, $n=2,3,6,9$, and 12) are commercially available and are commonly used ${ }^{47-51}$ as fluorescent probes. This fact opens up the possibility of measuring water concentrations at different mean radial positions in the micelle, using fluorescence and NMR techniques.

Acknowledgment. This work was supported by the Instituto Nacional de Investigaçao Cientifica. A.L.M. expresses his gratitude to the following entites, which made possible the construction of the single photon counting equipment: the Alexander-von-Humboldt Stiftung for the major financial support, Drs. R. Busse and K. A. Zachariasse for the information necessary to replicate their own SPC instrumentation, and the MaxPlanck-Institut fur biophysikalische Chemie (Gottingen) for the construction of some parts of the equipment. George Striker is gratefully acknowledged for making his deconvolution program available for us. Prof. R. Becker is also acknowledged for a critical reading of the manuscript.

\section{Appendix}

When steps 10 and 11 are included in Scheme I, the analytical concentration of water, $\left[\mathrm{H}_{2} \mathrm{O}\right]_{0}$, is related to the concentration of monomeric water, $\left[\mathrm{H}_{2} \mathrm{O}\right]$, and dimeric water, $\left[\left(\mathrm{H}_{2} \mathrm{O}\right)_{2}\right]$, by

$$
\begin{gathered}
{\left[\mathrm{H}_{2} \mathrm{O}\right]_{0}=\left[\mathrm{H}_{2} \mathrm{O}\right]+2\left[\left(\mathrm{H}_{2} \mathrm{O}\right)_{2}\right]} \\
=\left[\mathrm{H}_{2} \mathrm{O}\right]+2 K_{\text {dim }}\left[\mathrm{H}_{2} \mathrm{O}\right]^{2}
\end{gathered}
$$

which can be rearranged to give eq 12 .

Therefore, eq 3 will read

$$
\begin{gathered}
\tau_{0} / \tau=1+k_{\mathrm{a}} \tau_{0}\left[\mathrm{H}_{2} \mathrm{O}\right]+k_{\mathrm{a}}{ }^{\operatorname{dim}} \tau_{0}\left[\left(\mathrm{H}_{2} \mathrm{O}\right)_{2}\right] \\
\left\{\begin{array}{c}
k_{\mathrm{a}} \frac{\left(1+8 K_{\mathrm{dim}}\left[\mathrm{H}_{2} \mathrm{O}\right]_{0}\right)^{1 / 2}-1}{4 K_{\mathrm{dim}}}+ \\
k_{\mathrm{a}} \operatorname{dim} \frac{\left[\left(1+8 K_{\mathrm{dim}}\left[\mathrm{H}_{2} \mathrm{O}\right]_{0}\right)^{1 / 2}-1\right]^{2}}{16 K_{\mathrm{dim}}}
\end{array}\right\}
\end{gathered}
$$

Registry No. SDS, 151-21-3; DTAC, 112-00-5; $\mathrm{H}_{2} \mathrm{O}, 7732-18-5$; Triton X-100, 9002-93-1; methyl 9-anthroate, 1504-39-8.

(47) Blatt, E.; Ghiggino, K. P.; Sawyer, W. J. Chem. Soc., Faraday Trans. $11981,77,2551$.

(48) Vanderkooi, J.; Fishkoff, S.; Chance, B.; Cooper, R. A. Biochemistry $1974,13,1589$.

(49) Bashford, C. L.; Morgan, C. G.; Radda, G. K. Biochim. Biophys. Acta 1976, 426, 157

(50) Cadenhead, D. A.; Kellner, B. M. J.; Papahadjopoulos, D. Biochemistry $1977,16,5386$.

(51) Gabrini, G.; Verkman, A. S. Biochim. Biophys. Acta 1986, 862, 285. 